\title{
Cuidado nutricional hospitalar: percepção de nutricionistas para atendimento humanizado
}

\author{
Hospital nutritional care: \\ perception of the nutritionist for humanized attendance
}

Cassiani Gotâma Tasca Pedroso ${ }^{1}$

Anete Araújo de Sousa ${ }^{2}$

Raquel Kuerten deSalles ${ }^{2}$

${ }^{1}$ Universidade do Sul de Santa Catarina. Av. Pedra Branca 25, Cidade Universitária Pedra Branca. 88137-270 Palhoça SC. cassianigotama@yahoo.com.br ${ }^{2}$ Departamento deN utrição, Centro de Ciências da Saúde, U niversidadeFederal deSantaCatarina.
Abstract The research analyzed the actions of alimentary and nutritional care considering the perspectives of the nutritionists in a hospital reference for the $\mathrm{N}$ ational Politics of $\mathrm{H}$ umanization (PNH). From a qualitative approach, a focal group technique was used. The nutritionists were divided in two groups by working time, following homogeneity criteria. The interviews were developed for analysis of the category: Being a nutritionist for a humanized assistance, seeking to understand these professionals following actions: nutritional evaluation of the patient; planning implementation and evaluation of thenutritional and alimentary care. The analysis of the content was used as a techniquefor the systematization of the collected information grouped in units of meaning. The study disclosed that there is prioritization of the individualized assistance in function of the number of beds and bureaucratic activities, lack of autonomy in relation to the pre scription of diets, difficulties of interaction with other health professionals and between the nutritionists of the clinical and meal production areas. The results will provide the professional subsidies that substantiate actions for the construction of a model of humanized alimentary and nutritional care on hospitals.

Key words Collective feeding, Humanization of the assistance, Nutritionist, Nutrition services, Focal group
Resumo A pesquisa identificou as ações de cuidado alimentar e nutricional considerando as perspectivas da equipe de nutricionistas de um hospital de referência para a Política Nacional de Humanização (PNH). A partir de uma abordagem qualitativa, utilizou-se a técnica de grupos focais. A pesquisa envolveu treze nutricionistas, que foram divididas em dois grupos deacordo com o tempo de serviço, seguindo critérios de homogeneidade. As entrevistas foram desenvolvidas para análise da categoria: ser nutricionista para um atendimento humanizado, buscando-se conhecer as seguintes ações da prática destes profissionais: avaliação nutricional do paciente; planejamento, implementação e avaliação do cuidado nutricional e alimentar. A análise do conteúdo foi utilizada como técnica para sistematização das informações obtidas, agrupando-as em unidades de significado. 0 estudo revel ou que há priorização do atendimento individualizado em função do número de leitos e de atividades burocráticas, falta de autonomia em relação à prescrição de dietas, dificuldades de interação com outros profissionais de saúde e entre as nutricionistas das áreas clínica e produção de re feições. Os resultados deste estudo poderão oferecer aos profissionais subsídios quefundamentem ações para a construção de um modelo de cuidado alimentar e nutricional humanizado em hospitais. Palavras-chave Alimentação coletiva, Humanização da assistência, N utricionista, Serviços denutrição, Grupo focal 


\section{Introdução}

A Política Nacional de Humanização (PNH) em seu projeto destaca a necessidade de construir e implementar ações específicas para os diferentes setores de saúde, a fim de responder às necessidades da população de forma integrada à rede de serviços de saúde local e regional ${ }^{1}$.

Aliado à necessidade desta nova cultura de atenção, os novos perfis demográficos, de mortalidade e morbidade da população ${ }^{2}$ têm exigido abordagens diferenciadas para um atendimento qualificado. Ao lado destas demandas que afetam diretamente 0 atendimento nos hospitais, a desnutrição intra-hospitalar também é considerada um aspecto preocupante por estudiosos do tema $a^{3-6}$.

Diante deste contexto, o cuidado nutricional assumefundamental importância dentro do processo de humanização no ambiente hospitalar, visto que são poucos os indicadores e ações humanizadoras concebidos com os setores envolvidos neste cuidado. 0 termo cuidado é tradicionalmente adotado pela área de enfermagem. Borestein et al. ${ }^{7}$, ampliando este conceito para a área de saúde, destacam que o cuidado é um encontro estabelecido entre a pessoa que cuida e quem é cuidado, manifestando-se através de ações profissionais disciplinares e interdisciplinares que se dão no processo de interação terapêutica entre os seres humanos.

$\mathrm{N}$ a nutrição, a American Dietetic Association $(A D A)^{8}$ define 0 cuidado nutricional como um processo de ir ao encontro das diferentes necessidades nutricionais de uma pessoa, o que inclui [...] a avaliação do estado nutricional do indivíduo, a identificação das necessi dades ou problemas nutricionais, o planejamento de objetivos de cuidado nutricional que preencham essas necessidades, a implementação de atividades nutricionais [...] e a avaliação do cuidado nutricional. Ainda Boog ${ }^{9}$ destaca a necessi dade de envolver aspectos sensoriais e psicológicos ao cuidado nutricional.

Sousa e Proença ${ }^{10}$ adotam os termos cuidado alimentar e nutricional e argumentam que, para a efetivação destes cuidados, énecessário um conjunto de ações articuladas entreos setores de produção de refeições e de atendimento clínico-nutricional. Estas ações consistem em estratégias coletivas que visam não só às ações qualificadas em saúde e nutrição, como também à realização dos profissionais envolvidos pela oportunidade de interação e aprendizagem mútuas no trabaIho. Para o presente estudo, embora estes termos não sejam adotados pela legislação que regulamenta o exercício profissional do nutricio- nista no Brasil, os conceitos apresentados podem auxiliar nas práticas deste profissional dentro do contexto da humanização.

A inserção dos nutricionistas nos hospitais é destacada em pesquisa do Conselho Federal de Nutricionistas (CFN). Dos nutricionistas que atuam em nutrição clínica, cerca de $80 \%$ estão nos hospitais e clínicas e, dos que atuam na alimentação coletiva, $28 \%$ o fazem no setor hospitalar ${ }^{11}$. Em Santa Catarina, 48,4\% dos nutricionistas estavam no setor hospitalar ${ }^{12}$, havendo, posteriormente, uma migração para a área de alimentação coletiva, que engloba também outros setores, além do hospitalar ${ }^{13}$.

Além dos aspectos referidos, a situação do trabalho deste profissional tem sido objeto de alguns estudos. Os resultados têm revelado falta de comunicação entre as unidades de internação clínica e a produção de refeições, dificultando a interação entrea prescrição ea execução da dieta, bem como a análise da efetividade do tratamento nutricional oferecido ${ }^{10,14,15}$. Destaca-se ainda a falta de definição clara de responsabilidades, formação insuficiente dos profissionais de saúde para lidar com os problemas nutricionais, pouca influência sobre a adesão do paciente ao tratamento, falta de cooperação entre a equipe e de envolvimento da gerência dos hospitais ${ }^{16}$, além de acúmulo de atividades ligadas ao gerenciamento de serviços ${ }^{17}$.

Estes dados são relevantes para uma reflexão sobre as competências e habilidades que estes profissionais necessitam para o desenvolvimento de ações em alimentação e nutrição qualificadas dentro do contexto de humanização. 0 desenvolvimento destas ações depende de condições dignas de trabal ho e da interação de profissionais com os cuidados ao paciente.

A PNH é uma política generalista. Portanto, não apresenta especifici dades de ações relacionadas ao cuidado alimentar e nutricional. Baseado nestas considerações, o objetivo do estudo foi identificar as ações de cuidado alimentar enutricional, considerando a perspectiva de nutricionistas de um hospital de referência para a PN H.

\section{Percurso metodológico}

A pesquisa apresentou uma abordagem qualitativa. Para M inayo e Sanches ${ }^{18}$, este tipo de abordagem realiza uma aproximação fundamental e de intimidade entre sujeito e objeto, se envolvendo com empatia aos motivos, às intenções, aos projetos dos atores, a partir dos quais as ações, as estruturas e as relações tornam-se significativas. 
Considerando as características da pesquisa qualitativa e as contribuições para as ciências da saúde advindas dos estudos realizados com grupos $^{19,20}$, optou-se, para o presente estudo, pela utilização da técnica do grupo focal.

0 grupo focal é uma técnica de coleta de dados para pesquisa qualitativa inspirada em técnicas de entrevistas não direcionadas e técnicas grupais utilizadas na psiquiatria. Consiste em entrevistas em grupo, sobre um "foco" ou "tópico" específico. Busca colher informações quepossam proporcionar a compreensão de percepções, crenças, atitudes sobreum tema, produto ou serviço. Difere da entrevista individual por basearse na interação entre as pessoas para obter dados necessários à pesquisa. Sua formação deve obedecer a critérios previamente determinados pelo pesquisador, de acordo com os objetivos da investigação, cabendo a essea criação de um ambiente favorável à discussão, que propicie aos participantes manifestar suas percepções e pontos de vista ${ }^{21,22}$.

0 presente estudo foi desenvolvido no período de abril a agosto de 2006, com a equipe de nutricionistas que atua em um hospital público no município de Florianópolis (SC).

O hospital, referência na PN H , apresenta 260 leitos exclusivos do Sistema Ú nico de Saúde e dentre as ações que integram a Política de Humanização interna do hospital destacam-se 22 programas voltados para os trabalhadores da instituição e 42 programas e ações voltados aos usuários do sistema de saúde.

A equipe de nutricionistas é composta por treze profissionais, do sexo feminino. Atuam em diferentes setores: uma na gerência geral da unidade dealimentação en nutrição, duas na unidade de produção de refeições e dez nas unidades de internação clínica e ambulatório .

0 referencial teórico $0^{6,10,13}$ possibilitou a elaboração de um modelo de análise ${ }^{19}$ abordandose aspectos conceituais, práticas e éticas da assistência prestada aos pacientes, culminando na categoria "Ser nutricionista para um atendimento humanizado". A partir da pergunta norteadora, "O que é ser nutricionista para um atendimento humanizado?", buscou-secompreender as seguintes ações da prática destes profissionais (1) avaliação do paciente: aspectos clínicos relacionados ao histórico clínico nutricional; (2) planejamento do cuidado nutricional: aspectos clínicos referentes à prescrição dietética, bem como medidas que possibilitem ao pacienteatingir suasnecessidades nutricionais; (3) implementação do cuidado nutricional: aspectos relacionados à produção de refeições, modificações necessárias para tornar o alimento aceitável pelo pacienteeeducação nutricional ao paciente e à família e (4) avaliação do cuidado nutricional: aspectos relacionados ao monitoramento da ingestão alimentar, alteração do comportamento alimentar ao monitoramento dos dados clínicos e nutricionais.

Astrezenutricionistas foram divididas em dois grupos, estabelecendo-se como critério o tempo deserviço no hospital, respeitando-seas recomendações de homogeneidade e número de participantes ${ }^{23}$. O Grupo 1 foi composto por seis nutricionistas, que trabalham há mais de nove anos no hospital, enquanto o Grupo 2 foi composto por sete nutricionistas que trabalham há menos de nove anos. Foram realizados quatro encontros de discussão, sendo dois com cada grupo, com duração aproximada de uma hora e trinta minutos, cujo horário e local foram acordados entre as participantes. Cada encontro teve um objetivo euma técnica pré estabelecidos ${ }^{24}$. Ao início de cada reunião, utilizou-se música ambiente e solicitou-se às entrevistadas que fechassem os olhos, aplicando-se um exercício de respiração, para proporcionar relaxamento e concentração. Para as entrevistas, foi utilizada a técnica da "explosão de idéias", a partir da pergunta norteadora. As entrevistas foram gravadas e registradas em caderno de campo, sendo posteriormente transcritas ${ }^{25}$. A pós a transcrição, realizou-se a autoconfrontação, através da qual cada participante recebeu uma cópia da entrevista para acrescentar ou retirar algo ao material e assiná-lo.

A análise do conteúdo $(A C)^{26}$ foi utilizada como técnica para sistematização das informações obtidas, agrupando-as em unidades de significado. Posteriormente, o conteúdo foi analisado e discutido com o auxílio do referencial teórico sobre o tema.

0 projeto da pesquisa foi aprovado pelo ComitêdeÉtica de Pesquisa com Seres Humanos (CEPSH) da Universidade Federal de Santa Catarina (UFSC). A fim de assegurar 0 anonimato das participantes, foram utilizadas as letras "Nut", seguida por al garismos cardinais (Ex: N ut 1; N ut 2), durante a descrição das narrativas nos resultados e discussão.

\section{Resultados ediscussão}

A equipe de nutricionistas, com relação ao tempo de formação, é composta por três profissionais com menos de dez anos de formação, seis estão na faixa de dez a 21 anos e quatro há mais de vinte anos. Quanto ao aprimoramento profissional, seis profissionais realizaram especiali- 
zação na área de nutrição clínica, uma em administração, uma em gerência da qualidade deprodução de refeições, uma em gerontologia, uma em saúde pública e uma em terapia nutricional. Deste grupo, quatro são mestres, uma é mestranda euma doutoranda. 0 regime de trabalho é de trinta horas semanais, variando para as chefias que totalizam quarenta horas.

A análise do conteúdo revelou que, apesar das nutricionistasterem preocupação com a qualidade do cuidado prestado ao paciente, há predomínio de ações fragmentadas, não sistematizadas e dificuldades de interação com a equipe.

A avaliação clínico-nutricional éuma das primeiras ações realizadas na prática dos nutricionistas e faz parte do protocolo de atendimento do hospital. Ocorre, geralmente, nas primeiras horas de internação do paciente. Observam-se, primeiramente, os dados clínicos, bioquímicos, antropométricos e sociais, registrados pela equipe de enfermagem no prontuário. A história de vida dos pacientes foi um dos aspectos ressaltados no processo de avaliação: Eu sempre procuro ler o prontuário primeiro, saber por que o paciente está internando, de onde ele veio antes de falar com ele. Aí a gente conversa com o paciente já sabendo mais ou menos o que ele tem e o que não tem. (Nut 7)

A avaliação do estado nutricional tem como objetivo identificar os distúrbios nutricionais, possibilitando uma intervenção adequada para auxiliar na recuperação ou manutenção do estado de saúde do indivíduo. Utilizam-se métodos objetivos (antropometria, composição corpórea, parâmetros bioquímicos e consumo alimentar) e métodos subjetivos (exame físico a Avaliação Subjetiva Global), recomendando-se a associação destes indicadores para obter um adequado diagnóstico nutricional ${ }^{27}$.

Estas ações, quando realizadas conjuntamente pela equipe de saúde, podem auxiliar no cuidado nutricional, naevolução clínicaeprevenção da desnutrição hospitalar. Os conhecimentos em nutrição, a clareza de papéis e as responsabilidades da equipe são imprescindíveis neste processo ${ }^{28}$.

Destaca-se que a competência técnico-científica também faz parte da humanização. A PNH, além da incorporação de novas tecnologias e a especialização dos saberes, visa como princípio básico ao fortalecimento do trabalho em equipe, fomentando a multidisciplinaridade ${ }^{1}$.

Há uma preocupação das nutricionistas em utilizarem os dados obtidos pela equipe de enfermagem: Quando o pacientevem da enfermaria normalmentea enfermagem recebe ele ejá pesa emede, só queàs vezes elessó perguntam pro paciente. Quan- do você vai ver os dados não batem, porque foi um dado referido. Às vezes a pessoa não se pesa enão se mede há muito tempo edá diferença. (Nut 7)

É importante que as medidas antropométricas sejam coletadas pela equipe de saúde, pois quando referidas pelo paciente nem sempre são fidedignas. Wyszynski et al. ${ }^{29}$, investigando o estado nutricional de pacientes hospitalizados na Argentina, constataram que somente em $38,8 \%$ dos hospitais estudados houve registros sobre 0 estado nutricional e apenas $12 \%$ dos pacientes lembravam de seu peso usual e atual.

Com relação à obtenção dos dados bioquímicos, observam-se dificuldades para estabele cer parcerias entre médicos e nutricionistas. Como exemplo, as nutricionistas referem queos exames frequentemente solicitados pelos médicos e disponíveis no prontuário são hemograma, proteína total e albumina. Outros exames são solicitados somente em casos específicos: Têm médicos que aceitam nossos pedidos, outros não. N ormalmenteos médicos maisjovens ou mais abertos, que sabem a importância, aceitam meIhor. (Nut. 9)

A pós a observação do prontuário, as nutricionistas procuram saber como o paciente está e como se sente em relação à doença, objetivando a adequação da dieta às suas necessidades alimentares enutricionais e a sua condição de vida.

Os dados sociais também compõem as informações. É perguntado ao paciente se já esteve internado, se é portador de doença crônica ou se possui acompanhante. Para verificar o consumo alimentar per capita, especialmente de açúcar e óleo, costuma-se perguntar se mora sozinho ou com outras pessoas ou mesmo se é morador de rua: Quando eu vou conversar com ele, no primeiro momento eu faço algumas perguntas, como ele está como está se sentindo, como é a relação dele com o alimento, o que ele costuma comer, o que gosta e o que não gosta, o que aceita ou não aceita, como éque estava se alimentando em casa, se estava se alimentando ou não, pra ter uma idéia da disponibilidade de alimentos na casa dele, procuro perceber como ele está em relação à doença dele, se ele sabe realmente o que ele tem. (Nut 6)

Para o registro das informações, é utilizado um formulário específico (histórico denutrição), usualmente anexado ao prontuário do paciente, constando de dados gerais, história alimentar, análise dietética, avaliação nutricional e conduta dietoterápica. Porém, as profissionais afirmam que o formulário é extenso, tornando-se de difícil aplicação, considerando o número de pacientes por nutricionista e o tempo necessário para a sua aplicação: Quando a gente está com trinta leitos, sem 
condições de fazer histórico, pelo menos colhemos as informações principais, para auxiliar na adequação da dieta. N ós temos o formulário oficial, que deveríamos aplicar em todos os pacientes, mas é muito grande, toma muito tempo, como a gente normalmente é uma para trinta leitos, não dá. (Nut 1)

No Canadá, Labonté e Ouelét ${ }^{14}$ analisaram o tempo necessário para os dietistas (título equivalente ao nutricionista no Brasil) desenvolverem suas atividades em hospitais e constataram que os dietistas dedicam pouco tempo ao preenchimento de formulários contendo dados clínicos nutricionais do paciente.

No Brasil, a relação entreo número de nutricionistas e o número de leitos foi estabelecida pela Resolução no 201/98 do Conselho Federal de N utricionistas, que recomenda uma nutricionista para cinquenta, trinta ou quinzeleitos, conformeo nível decomplexidade da assistência prestada ao paciente ${ }^{30}$.

$\mathrm{Na}$ França, Guy-Grand ${ }^{31}$ revelou insuficiência deprofissionaiseumagrandeheterogeneidadena proporção de dietistas/número de leitos. A média encontrada foi de 1 dietista /117 leitos. No Brasil, em estudo comparando-se um hospital público e outro privado, constatou-se a relação de 1 nutricionista/36 e 44 leitos, respectivamente ${ }^{32}$.

A priorização do atendimento e a necessidade de reformulação das ações de trabalho são relatadas pelas profissionais, considerando os limites apresentados: A gente visita a todos, mas avalia aqueles que a gente julga mais graves, que precisam de maior atenção. Pra isso, não tem padronização ainda. Então, cada um na clínica vê 0 paciente que é mais grave. A gente prioriza. $0 \mathrm{~s}$ outros, você visita, mas dependendo da patologia ou até mesmo de como ele está evoluindo, não é necessária uma avaliação diária. (N ut 6)

O histórico nutricional e as orientações dietoterápicas para a alta têm sido objeto de reformulação. No que diz respeito ao histórico nutricional, a proposta baseia-se na aplicação de um instrumento para agilização da avaliação nutricional, a avaliação subjetiva global (ASG) ${ }^{33}$, no intuito de otimizar o cuidado nutricional.

Aquino ${ }^{34}$ destaca a importância da realização da triagem nutricional e afirma queé o primeiro passo para 0 atendimento ao indivíduo hospitalizado eseu desenvolvimento deveser realizado a partir da identificação dos fatores associados ao risco de desnutrição na população.

A partir das informações que compõem a avaliação clínico-nutricional, o processo de planejamento e implementação do cuidado nutricional, referido pela equipe, objetiva prescrever uma dieta de acordo com as necessidades ali- mentares enutricionais dos pacientes: 0 planejamento ocorre de imediato, quando você conhece o paciente. $\mathrm{N}$ em todo o paci ente necessita de cálculo denecessi dades. Por exemplo, uma ci rurgia de hérnia, se o paciente não for diabético ou hipertenso, éuma dieta normal. Já um paciente que já traz no diagnóstico disfagia por alimentos sólidos, aí eu tenho que fazer um trabalho melhor. Faço os cálculos por $\mathrm{H}$ arris Benedict (fator injúria e atividade), acompanho a ingestão, calculo, peso diariamente. (Nut 8)

A prescrição da dieta é realizada pelos médicos. H aven do necessidade de al teraçõ̃es, as nutricionistas comunicam-se com o médico responsável. Entretanto, algumas profissionais, sobretudo as com menos tempo de serviço, relataram dificuldades de parceria com os médicos, destacando que al guns não aderem ao trabalho multidisciplinar. Revelaram, ainda, sentir insegurança com relação à autonomia das suas ações: Eu procuro conversar, mesmo sem ter muita abertura, registro no prontuário o que eu acho, mas nem sempre sou atendida, e, muitas vezes, fica como está. Até porque eu não posso tomar nenhuma atitude, modificar sozinha. Não sei atéquando isso é corre to, não sei até onde vai nossa autonomia. (Nut 7)

Boog $^{35}$, em estudo sobre as dificuldades encontradas por médicos e enfermeiros na abordagem de problemas alimentares, considera que o nutricionista é habilitado por formação para adequar as orientações alimentares à realidade dos pacientes. De fato, esta ação é reconhecida pela legislação que rege a profissão ${ }^{30} \mathrm{e}$ implica, necessariamente, a conjugação de saberes de nutricionistas, enfermeiros, médicos e outros profissionais da equipe.

Outro aspecto destacado pela equipe está relacionado ao encaminhamento das alterações de dietas para o setor de produção de refeições: 0 ficialmente, as alterações do dia começam a vigorar a partir do caféda tarde, só que quando se trata de uma dieta de evolução, e a gente vê que já dá pra fazer na hora do almoço, a gente já fala pra copeira, não espera chegar a tarde. Então isso às vezes é pra tentar adiantar o serviço. Por exemplo, a dieta era líquida restrita e já foi liberada líquida completa, porque eu vou esperar até a hora do lanche, sea gentevêqueelejá pode comer melhor? (Nut 1)

Se o paciente estava em dieta zero e já pode comer, a gente não vai esperar até a tarde pra dar comida pra ele. (Nut 2)

Embora a solicitação de alteração e a liberação de dieta sejam operacionalizadas antecipadamente, visando melhorar a aceitação da alimentação pelo paciente, podem-se acarretar dificuldades para o setor de produção: Têm coisas que as 
copeiras pedem na hora do almoço, mas não dá tempo de descongelar, daí fica para o jantar. M as elastambém perdem os pedidos. Às vezes, chega pedido de alteração às onze da manhã enessa hora já está tudo feito, não tem como mudar. (Nut 4)

Mas, se por um lado há uma preocupação com a adaptação da dieta, o gerenciamento do tempo destas atividades gera distanciamento de outras ações também relacionadas com a atenção ao paciente, conforme relatado pelas nutricionistas das unidades de internação clínica:

N ão dá tempo pra acompanhar todos os pacientes. Há muito pra fazer, ver sonda, prontuários, registrar, conversar com médicos, copeiras, orientações de alta. (Nut 9)

Tem que supervisionar copeira, fazer atividades burocráticas aqui embaixo [na produção de refeições] emuitas vezes não dá tempo, fora as coberturas extras que a gente tem quefazer, sempretem alguém defolga ou férias, emergência, enfim! (Nut 7)

Beck et al. ${ }^{16}$ evidenciam um distanciamento deste profissional dos aspectos técnicos e educacionais em favor de conhecimentos administrativos egerenciais.

O nutricionista, tanto nas instituições hospitalares quanto em outros setores, lida com situações de trabal ho diversificadas e com diferentes graus de complexidade, demandando destes profissionais exigências cognitivas e psíquicas ${ }^{10,36}$.

Com relação à individualização da dieta, este aspecto parece ser um desafio para os profissionais envolvidos com a produção das refeições. Há dificuldades dese perceber o pacienteno processo produtivo, pois o fluxo dos alimentos segue a lógica da padronização e da divisão do trabalho ${ }^{10}$ : D efinido qual énosso plano dietoterápico, coloca-se no mapa de dietas, encaminhamos para a copeira. Como o cardápio é padronizado e é trabalhado com médias por tipo de dieta, aquelepacientevai entrar pra produção no rol das dietas brandas, pastosas. (N ut 1 )

O cardápio segue um padrão dentro da alimentação normal e dentro dele tem as preparações que são feitas de acordo com pedidos extras, pelas preferências ou necessidades deles. A gente percebe ali embaixo [na produção de refeições] quetem pessoas diferentesinternadas quando tem pedidos diferentes, se não a gente olha e é tudo igual. Por exemplo, uma dieta branda é toda igual, você não vê uma pessoa, esim um monte de dietas. A não ser quando tem o pirão ou o peixe frito do Sr. João. (Nut 4)

A humanização também écaracterizada pelo atendimento destasindividualidades, considerando que a inapetência ou a rejeição aos alimentos pode comprometer o tratamento ${ }^{20}$. Desta forma, é fundamental considerar que 0 ato de se alimentar é parte da construção de identidades culturais e sociais e envolve emoção, memória e sentimentos, que refletem a qualidade simbólica do alimento ${ }^{37}$.

$\mathrm{Na}$ instituição, as nutricionistas consideraram que, apesar de ser um hospital público, e apresentar restrições financeiras, existe grande variedade de alimentos disponíveis. $\mathrm{Na}$ falta de determinado produto, as profissionais comunicam-se com a família do paciente ou com a direção do hospital no intuito de obtê-lo, buscando desta forma a individualização do atendimento.

0 acompanhamento da aceitação da dieta é uma das ações consideradas importantes dentro do processo de avaliação do cuidado alimentar e nutricional. Através do acompanhamento, épossível identificar alterações a serem realizadas, dependendo das necessidades nutricionais (redução ou aumento da oferta de nutrientes), prefe rências ou aversões alimentares do paciente: Estaremosacompanhando se isso deu certo, se a aceitação éboa, emesmo que seja boa, seprecisa acrescentar algum suplemento, tirar alguma coisa. Às vezes têm pacientes obesos que não estão internados pra fazer dieta de emagrecimento, mas a gente reduz as calorias necessárias. Aí é o acompanhamento do dia a dia queéessa visita diária. (Nut 1)

Se o paciente tem algum grau de desnutrição ou está caminhando pra isso, eu entro com suplemento, modifico alguma preparação, aumento a oferta calórica. (Nut 8)

Entretanto, para analisar a efetividade do tratamento e, dessa forma, avaliar sua própria conduta, hánecessidade derealizar a avaliação continuada para controle mais racional do tratamento erecuperação do estado de saúdee nutrição do paciente ${ }^{38}$. No entanto, para as nutricionistas, 0 sucesso do tratamento, depende de outros fatores: A avaliação está num conjunto de coisas que a gentefaz, desdeo início do tratamento. 0 sucesso do tratamento, ou o cumprimento do objetivo, também depende de outros fatores, que não só nós, porqueentrea informação sair lá decima [unidade de internação] e passar para a cozinha, nesse meio campo, aí acontece muita coisa. (Nut 1)

0 planejamento e o tratamento caminham juntos com a produção. Não adianta você saber tudo sobre o paciente, as preferências, aversões, se vocênão passa isso pra frente. (Nut 8)

Alguns estudos ${ }^{38,39}$ têm destacado que a aceitação da alimentação por parte do paciente internado envolve aspectos relacionados à patologia, às características das refei ções of erecidas eao ambiente. Além destes aspectos, a aceitação da alimentação está relacionada aos profissionais envolvidos no cuidado alimentar enutricional. 


\section{Considerações finais}

As principais dificuldades encontradas pelas nutricionistas relacionam fragmentação de ações entre os profissionais da equipe, além de condições geradas pela própria complexidade de suas atividades, tais como tempo escasso, número de leitos por nutricionista e atividades administrativas. Possivelmente, a sistematização das ações de cuidado alimentar e nutricional conjugada a um protocolo de triagem possa otimizar a organização do trabalho destes profissionais.

Outro aspecto observado refere-se à falta de interação entreas nutricionistas das áreas clínica e produção de refeições, uma vez que estas últimas parecem não se sentir integrantes no processo de cuidados do paciente.

0 cuidado nutricional envolvetanto a avaliação do estado nutricional, a identificação de necessidades nutricionais, o planejamento, implementação e avaliação do cuidado, como também os processos da compra, armazenamento, higienização, pré-preparo e preparo, porciona-

\section{Colaboradores}

CGT Pedroso trabalhou em todas as etapas da pesquisa, desde a concepção do projeto e coleta de dados até a elaboração do artigo; AA Sousa orientou todas as etapas da pesquisa, participou da análise da pesquisa, revisão do texto final, idealização e elaboração do artigo e RK Salles orientou o delineamento metodológico, a análise dos dados e a elaboração do artigo. mento, transporte e apresentação final das preparações. Assim, as duas formas de atuação são interdependentes, pois considerá-las de forma isolada seria reduzir a complexidade do cuidado ao paciente, que deve considerar a comida em seus aspectos simbólicos e sensoriais.

A dificuldade de relacionamento com os outros profissionais de saúde também ficou constatada durante o processo de avaliação nutricional. Esta dificuldadeéobservada especial mentecom a equipe médica, no que diz respeito às modificações da prescrição dietética e solicitação de exames laboratoriais. A partir dos relatos, observouse a carência de uma formação de profissionais da saúde baseada na interdisciplinaridade.

Os resultados encontrados neste trabalho poderão oferecer aos profissionais subsídios quefundamentem suas ações para a construção de um modelo decuidado alimentar enutricional humanizado em hospitais. Além disso, a reprodução de estudos sobre este tema em diferentes contextos pode contribuir ao aprofundamento conceitual acerca do cuidado em alimentação enutrição.

\section{Referências}

1. Brasil. Ministério da Saúde. Política Nacional de H umanização (H umaniza-Sus). 2005. [site da Internet] [acessado 2005 jun 17]. Disponível em: http:/ /www.portal.saude.gov.br

2. Chaimowicz F. A saúde dos idosos brasileiros às vésperas do século $X X I$ : problemas, projeções e alternativas. Rev. Saude Publica 1997; 31(2):184-200.

3. Waitzberg DL, Waleska T, Caiaffa MD, Correia ITD. Hospital malnutrition: the Brazilian National Survey (IBRANUTRI): A study of 4000 patients. Nutrition 2001; 17(7/8):553-580.

4. Mello ED, Beghetto M G, Teixeira LB, Luft VC. Desnutrição hospitalar cinco anos após o IBRANUTRI. Rev Bras Nutr Clin 2003; 18(2):1-10.

5. Gottraux S, Maisonneuve N, Gevaux D, FonzoChriste C, Chiki M, Guinot-Bourquin S, Vernet $P$, Metivier M F, Raguso C, Pichard C. Screening and treatment of malnutrition: European Council Resolution and its potential application in Switzerland. M ed Suisse Romande 2004; 124(10):617-623.

6. Merhi LVA, Garcia RWD, M ônaco DV, Olivera YMRM . Comparación del estado nutricional, consumo alimentício y tiempo de hospitalización de pacientes de dos hospitales, uno público e otro privado. Nutr Hosp 2006; 21(1):32-37.

7. Borenstein MS, Oliveira ME, Padilha MICS. Visualizando o cuidado de enfermagem por meio de imagens. Rev Cienc Saúde 2003; 22(1/2):32-39. 
8. American Dietetic Association. Identifying patients at risk: ADA's definitions for nutrition screening and nutritional assessment. J Am Diet Assoc 1994; 94(8):838-839.

9. Boog MCF. O papel do enfermeiro no cuidado nutricional ao paciente hospitalizado. Rev Campineira Enf. 1999; 2(1):17-21.

10. Sousa AA, Proença RPC. La gestion des soins nutritionnels dans le secteur hospitalier: une étude comparative Brésil-France. Rech Soins Infirm 2005; 83:2833.

11. Conselho Federal de Nutricionistas. Inserção Profissional dos Nutricionistas no Brasil. [site da Internet] [acessado 2008 nov 27]. Disponível em: http:// www.cfn.org.br/

12. Vasconcelos FAG. Um perfil do nutricionista em Florianópolis - Santa Catarina. Rev Ciênc Saúd 1991; 10(1/2):73-86

13. Alves E, Rossi CE, Vasconcelos FAG. Nutricionistas egressos da Universidade Federal de Santa Catarina: áreas de atuação, distribuição geográfica, índices de pós-graduação e de filiação aos órgãos de classe. Rev. Nutr. 2003; 16(3):295-304.

14. Labonté $L$, Ouellét $D$. Nutrition clinique, étude du temps consacré aux soins. Diététique en action 1996; 10:25-26.

15. Ouellét $D$, Pigeon J. Validation de l'unité de mesure en nutrtion clinique: Unité Podérée de $\mathrm{N}$ utrition $\mathrm{Cli}$ nique (UPNC) [rapport final]. Quebéc: Département dês sciences dês aliments et de nutrition, Faculté des sciences de l'agriculture et de l'alimentation; 1997.

16. Beck $A M$, Balknäs UN, Furst $P, H$ asunen $K$, Jones $L$, Keller U, M elchior JC, Mikkelsen BE, Schauder $P$, Sivonen $L$, Zinck $O$, Øien $H$, Ovesen $L$, Council of Europe (the Committee of Experts on Nutrition, Food Safety and Consumer $\mathrm{H}$ ealth of the Partial Agreement in the Social and Public Health Field) Food and nutritional care in hospitals: how to prevent undernutrition report and guidelines from the Council of Europe. Clin Nutr 2001; 20(5):455-460.

17. Ansaloni JA. Situação de trabalho dos nutricionistas em empresas de refeições coletivas de Minas Gerais: trabalho técnico, supervisão ou gerência? Rev. Nutr. 1999; 12(3):241-260.

18. M inayo M CS, Sanches O. Quantitativo-qualitativo: oposição ou complementaridade? Cad Saude Publica 1993; 9(3):237-248.

19. Sobal J. Sample extensiveness in qualitative nutrition education research. J N utr Educ 2001; 33(4):184192.

20. Flik U. U ma introdução à pesquisa qualitativa. $2^{2} \mathrm{ed}$. Porto Alegre: Bookman; 2004

21. M inayo M CS. 0 desafio do conhecimento: pesquisa qualitativa em saúde. 4ạ ed. São Paulo: Hucitec; Rio de Janeiro: Abrasco; 1996

22. Carlini-Cotrim B. Potencialidades da técnica qualitativa grupo focal em investigações sobre abuso de substâncias. Rev. Saude Publica 1996; 30(285):93.

23. Tanaka OY, M elo C. Avaliação de Programas de Saúde do Adolescente- um modo de fazer. São Paulo: Edusp; 2001.
24. Ressel LB, Gualda DM R, Gonzales RM B. Grupo focal como uma estratégia para coletar dados de pesquisa em enfermagem. Int J Qual M eth 2002; $1(2): 1-29$

25. Levy FM L, Matos PES, Tomita NE. Programa de agentes comunitários de saúde: a percepção de usuários e trabalhadores da saúde. Cad Saude Publica 2004; 20(1):197-203.

26. Bardin L. Análise de conteúdo. Lisboa: Edições 70 2004.

27. Cupari L. Guias de M edicina Ambulatorial e Hospitalar. Nutrição Clínica no Adulto. 2ª ed. São Paulo: Manole; 2005.

28. Campos SH, Boog M CF. Cuidado nutricional na visão de enfermeiras docentes. Rev. Nutr. 2006; 19(2):144-155

29. Wyszynski DF, Perman M, Criveli A. Prevalence of Hospital Malnutrition in Argentina: Preliminary Results of a Population-Based Study. Nutrition 2003; 19(2):115-119.

30. Conselho Federal de Nutricionistas. Definição de Atribuições Principal e Específicas dos Nutricionistas. Resolução no 200/98, de 8 mar 1998. Diário Oficial da União 1998; 20 mar.

31. Guy-Grand B. Alimentation en milieu hospitalier. Paris: Rapport de Mission; 1997.

32. Garcia RWD. A dieta hospitalar na perspectiva dos sujeitos envolvidos em sua produção e em seu planejamento. Rev. Nutr. 2006; 19(2):129-144.

33. Detsky AS, M CLaughlin JR, Baker JP, Johnston N, Whittaker S, M endelson RA, Jeejeebhoy KN. What is subjective global assessment of nutritional status? J Parenter Enteral Nutr 1987; 11(1):8-13.

34. Aquino RC. Fatores associados ao risco de desnutrição e desenvolvimento de instrumentos de triagem nutricional [tese]. São Paulo (SP): Universidade de São Paulo; 2005.

35. Boog MCF. Dificuldades encontradas por médicos e enfermeiros na abordagem de problemas alimentares. Rev. Nutr. 1999; 12(3):261-272.

36. Bosi M LM. Trabalho e subjetividade: cargas e sofrimento na prática da nutrição social. Rev. Nutr. 2000; 13(2):107-115.

37. Poulain JP, Proença RPC. O espaço social alimentar: um instrumento para o estudo dos modelos alimentares. Rev. Nutr. 2003; 16(13):245-256.

38. Oliveira AF, Oliveira FLC, Juliano Y, Ancona-Lopez F. Evolução nutricional de crianças hospitalizadas e sob acompanhamento nutricional. Rev. Nutr. 2005; 18(3):348.

39. Kandela P. Hospital food. Lancet 1999; 353(9154): 763.

Artigo apresentado em 26/02/2008

Aprovado em 26/11/2008

Versão final apresentada em 30/10/2008 